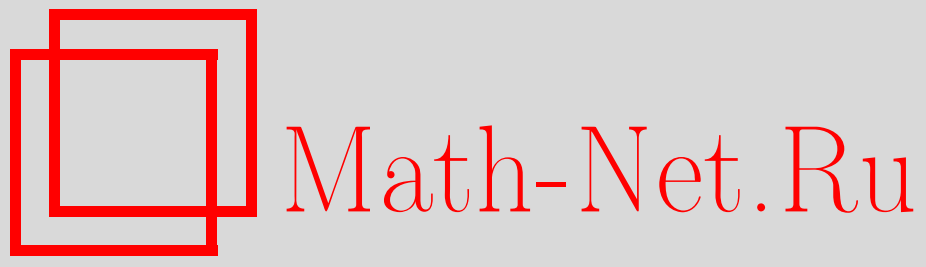

В. П. Паламодов, Интегралы Фурье, специальные функции и феномен полунепрерывности, Функи. анализ и его прил., 2001, том 35, выпуск 2, 53-63

DOI: https://doi.org/10.4213/faa245

Использование Общероссийского математического портала MathNet.Ru подразумевает, что вы прочитали и согласны с пользовательским соглашением

http://www . mathnet.ru/rus/agreement

Параметры загрузки:

IP : 35.173 .219 .149

26 апреля 2023 г., 14:24:15

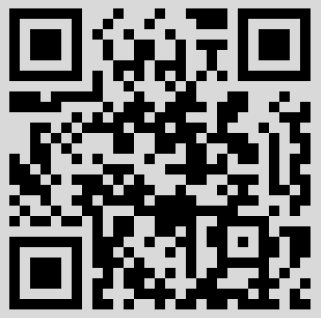




\title{
Интегралы Фурье, специальные функции и феномен полунепрерывности
}

\author{
(c) 2001. В. П. ПАЛАмОДОВ
}

\section{§1. Введение}

Со всякой эллиптической деформацией квазиоднородного ростка гиперповерхности связываются квазиоднородные специальные функции. Особенности этих функций характеризуются рациональными числами, которые называются энергетическими показателями. Применение вычетной формы к соответствующим интегралам Фурье приводит к интерпретации энергетических показателей в терминах геометрии лагранжева многообразия, порожденного деформацией. Мы приведем вычисления энергетических показателей для серии примеров и обсудим две гипотезы, в частности, феномен полунепрерывности при деформациях.

\section{§2. Интегралы Фурье и символы}

Запишем интеграл Фурье в виде

$$
I(\phi, a)=\int_{\Theta} \exp (2 \pi \imath \phi(x, \theta)) a(x, \theta) d \theta, \quad x \in X .
$$

Здесь $X$ - гладкое многообразие, а интегрирование ведется по открытому конусу $\Theta$ во вспомогательном пространстве $\mathbb{R}^{N} \backslash 0$ с координатами $\theta_{1}, \ldots, \theta_{N}$ и объемной плотностью $d \theta:=d \theta_{1} \wedge \cdots \wedge d \theta_{N}$. Группа $\mathbb{R}_{+}$положительных чисел действует в $X \times \Theta$ по правилу $t:(x, \theta) \mapsto(x, t \theta)$. Фаза $\phi$ есть вещественная гладкая однородная функция порядка 1 по отношению к этому действию и $d \phi \neq 0$. Амплитуда $a$ - также гладкая функция в $X \times \Theta$, причем предполагается, что образ ее носителя в пространстве $X \times \Theta / \mathbb{R}_{+}$имеет компактное замыкание. Мы предполагаем также, что амплитуда $a$ имеет структуру $a=a_{M}+r$, где $a_{M}$ есть гладкая однородная функция некоторой степени $M$. Остаток удовлетворяет оценкам $\left|D_{x}^{i} D_{\theta}^{j} r\right| \leqslant C_{i j}(x)(|\theta|+1)^{m-j}$ для некоторого $m<M$, где $C_{i j}$ суть непрерывные функции на $X$ для всех $i, j$, таких, что $i+j \leqslant J$, а число $J$ достаточно велико. Интеграл (2.1) определен как обобщенная функция в $X$ [2]. Выберем гладкую объемную плотность $d V$ в $X$ и рассмотрим обобщенную полуплотность $I(\phi, A)=I(\phi, a) \sqrt{d V}, A=a \sqrt{d V}$ (т. е. функционал на пространстве гладких полуплотностей $\rho$ с компактными носителями). Критические точки фазовой функции образуют множество $C(\phi)=\left\{\phi_{\theta}^{\prime}=0\right\} \subset X \times \Theta$. Это множество коническое, т.е. инвариантное относительно действия группы $\mathbb{R}_{+}$. Фазовая функция невырожденна, т.е. формы $d \phi_{\theta_{1}}^{\prime}, \ldots, d \phi_{\theta_{N}}^{\prime}$ линейно независимы на критическом множестве. Из этого условия следует, что $C(\phi)$ есть многообразие размерности $\operatorname{dim} X$. Пусть $T^{*}(X)$ - кокасательное расслоение и $T_{\circ}^{*}(X)-$ его подмножество 
ненулевых ковекторов. Отображение

$$
\tilde{\phi}: C(\phi) \rightarrow T_{\circ}^{*}(X), \quad(x, \theta) \mapsto\left(x, d_{x} \phi(x, \theta)\right),
$$

является иммерсией, образ $\Lambda(\phi)$ которой есть коническое лагранжево многообразие.

Возьмем копию $\Theta^{\circ}$ конуса $\Theta$ и введем два дифференциала $d=d_{X}+d_{\Theta}$ и $d^{\circ}=d_{\Theta^{\circ}}$ в комплексе де Рама $\Omega^{*}\left(X \times \Theta \times \Theta^{\circ}\right)$. Рассмотрим фазовую функцию $\Phi\left(x, \theta, \theta^{\circ}\right)=\phi\left(x, \theta+\theta^{\circ}\right)$ на $X \times \Theta \times \Theta^{\circ}$, а также подмногообразия $C^{\circ}(\Phi)=$ $\left\{d^{\circ} \Phi=0\right\}, Z=\left\{\theta^{\circ}=0\right\}$. Пересечение $C^{\circ}(\Phi) \cap Z$ изоморфно критическому множеству $C(\phi)$. Определим коориентацию на $C^{\circ}(\Phi) \cap Z$ с помощью 1 -форм

$$
d \Phi_{1}^{\prime}, \ldots, d \Phi_{N}^{\prime}, d \theta_{1}^{\circ}, \ldots, d \theta_{N}^{\circ}, \quad \text { где } \Phi_{i}^{\prime}:=\partial \Phi / \partial \theta_{i}^{\circ}, i=1, \ldots, N .
$$

Эта коориентация не зависит от выбора координат в $\Theta$. Произведение $\alpha:=d V \wedge$ $\left(d d^{\circ} \theta \theta^{\circ}\right)^{\wedge N}$, где $\theta \theta^{\circ}:=\sum \theta_{i} \theta_{i}^{\circ}$, есть гладкая плотность в $X \times \Theta \times \Theta^{\circ}$. Обозначим через $\rho$ любую форму, которая удовлетворяет уравнению $\left(-\imath d d^{\circ} \Phi\right)^{\wedge N} \wedge \rho=d V \wedge$ $\left(d d^{\circ} \theta \theta^{\circ}\right)^{\wedge N}$. Она определена с точностью до формы $\rho^{\prime}$, такой, что $\left(d d^{\circ} \Phi\right)^{\wedge N} \wedge \rho^{\prime}$ $=0$. Эта форма принадлежит идеалу в $\Omega^{*}\left(X \times \Theta \times \Theta^{\circ}\right)$, порожденному формами (2.2), ввиду невырожденности фазы $\phi$. Поэтому ограничение формы $\rho$ на многообразие $C^{\circ}(\Phi) \cap Z$ однозначно определено. Положим

$$
\sigma(I(\phi, A)):=a_{M} \sqrt{\rho \mid C(\Phi) \cap Z} .
$$

Это однородная полуплотность степени $M+N / 2$ на $C(\phi)$. Она называется символом интеграла Фурье $I=I(\phi, A)$. Если отображение $\tilde{\phi}: C(\phi) \rightarrow \Lambda(\phi)$ инъективно, мы определяем прямой образ символа. Он является однородной полуплотностью на $\Lambda(\phi)$ того же порядка. Число $M+N / 2$ называется степенью интеграла Фурье $I(\phi, A)$. Имеется неоднозначность в определении квадратного корня $S(\phi)$ в (2.3), которая несущественна в данном контексте. Глобальная конструкция изложена в [6].

ЗАмЕчАНИЕ. Абсолютное значение полуплотности (2.3) совпадает с главным символом в смысле [2] с точностью до множителя $(2 \pi)^{N / 2}$.

Контактный символ. Факторпространство $C^{*}(X)=T_{\circ}^{*}(X) / \mathbb{R}_{+}$есть многообразие коориентированных контактных элементов в $X$. Для конического лагранжева многообразия $\Lambda \subset T_{\circ}^{*}(X)$ множество $\Lambda_{c}=\Lambda / \mathbb{R}_{+} \subset C^{*}(X)$ есть многообразие размерности $\operatorname{dim} X-1$. Мы называем образ $L:=p\left(\Lambda_{c}\right)$, где $p: C^{*}(T) \rightarrow X$ - естественная проекция, локусом ${ }^{1)}$ лагранжева многообразия $\Lambda$. Для всякого интеграла Фурье $I=I(\phi, A)$ степени 0 мы задаем полуплотность

$$
\sigma_{c}(I):=\sqrt{\frac{|\theta|}{d|\theta|}} \sigma(I) .
$$

Правая часть однородна нулевой степени; следовательно, она корректно определена на $X \times \Theta / \mathbb{R}_{+}$. Образ этой полуплотности на $\Lambda_{c}$ называется контактныцм символом интеграла $I$.

1) Я предпочитаю слово «локус» стандартному термину «волновой фронт», который часто используется в другом смысле. 


\section{§3. Вычеты интегралов Фурье}

Для интеграла Фурье $I$ нулевой степени плотность $|I|^{2} d V$ имеет логарифмическую расходимость на локусе $L$ многообразия $\Lambda=\Lambda(\phi)$. Вычет логарифмического члена есть эрмитова форма со значениями в пространстве мер на $L$. Возьмем произвольную точку $\lambda \in \Lambda$ и запишем $\lambda=(y, h), h \in C_{y}^{*}(X)$. Выберем росток гладкой гиперповерхности $H \subset X$, который касается контактного элемента $h$, и рассмотрим гладкую ретракцию $q: Y \rightarrow H$ в некоторой окрестности $Y$ точки $y$. Обозначим через $m(\lambda)$ алгебраическую кратность отображения $q p$ в $\lambda$. Она не зависит от выбора гиперповерхности $H$ и ретракции $q$. Скажем, что лагранжево многообразие $\Lambda$ имеет конечную кратность над точкой $y$, если $m(\lambda)<\infty$ для каждой точки $\lambda \in \Lambda$ над $y$; мы полагаем $m(y):=\sum\{m(\lambda) ; p(\lambda)=y\}$. В этом случае существует вещественная гладкая функция $D$, которая обращается в нуль на локусе $L$ и удовлетворяет условиям

$$
d^{i} D(y)=0, \quad i<m, \quad d^{m} D(y) \neq 0, \quad m=m(y),
$$

где $d^{i}$ обозначает $i$-й дифференциал [6]. Любая функция $D$, наделенная этими свойствами, называется барьером лагранжева многообразия $\Lambda$ (и его локуса) в точке $y$. Если $\Lambda$ симметрично, т. е. инвариантно относительно инволюции $(x, \xi) \mapsto(x,-\xi)$, то существует барьер $D$, который удовлетворяет условиям $(3.1)$ с числом $m^{\prime}(y):=m(y) / 2$.

Теорема 3.1 [6]. Пусть $\Lambda$ - замкнутое симметричное лагранжево многообразие, имеющее конечную кратность над некоторой точкой $у \in X$. Для любых интегралов Фурье $U=I(\phi, A), V=I(\psi, B)$ нулевой степени, таких, что $\Lambda(\phi) \cup \Lambda(\psi) \subset \Lambda$, выполняется равенство

$$
\int_{D^{2} \geqslant \varepsilon^{2}} \alpha U \bar{V}=\left(\int_{\Lambda_{c}} \alpha \sigma_{c}(U) \bar{\sigma}_{c}(V)+o(1)\right) \log \frac{1}{\varepsilon}, \quad \varepsilon \rightarrow 0, \alpha \in \mathscr{D}(Y),
$$

где $D$ - барьер многообразия $\Lambda$ в у, а $Y$ - некоторая окрестность этой точки.

Распределение

$$
\Sigma_{\Lambda}[U, \bar{V}](\alpha):=\int_{\Lambda_{c}} \alpha \sigma_{c}(U) \bar{\sigma}_{c}(V)
$$

называется вычетом пары $U, V$. Символ $\Sigma_{\Lambda}$ есть эрмитова форма, значениями которой служат (комплекснозначные) меры на локусе $L$.

\section{§4. Деформации и специальные функции}

Пусть $f-$ голоморфная функция в открытом множестве $\Omega \subset \mathbb{C}^{\nu}$. Предположим, что начало координат $0 \in \Omega$ есть изолированная критическая точка функции $f$, причем $f(0)=0$. Рассмотрим росток $Z_{0}$ гиперповерхности $\{f=0\}$ в начале координат. Пусть $(S, 0)-$ росток комплексного пространства и $Z \subset S \times \Omega$ - гиперповерхность, заданная уравнением $F(s, \omega)=0$, где $F$ - некоторая голоморфная функция, причем $F(0, \omega)=f(\omega)$. Проекция $\pi: Z \rightarrow S$ есть деформация ростка $Z_{0}$. Обозначим через $O_{0}(\Omega)$ алгебру голоморфных функций на ростке $(\Omega, 0)$. Факторалгебра $T^{1}\left(Z_{0}\right)=O_{0} /\left(f, f_{\omega}^{\prime}\right)$ изоморфна пространству деформаций ростка $Z_{0}$ над двойной точкой. Размерность $\tau\left(Z_{0}\right):=\operatorname{dim} T^{1}\left(Z_{0}\right)$, называемая числом Тюриной, конечна. Отображение Кодаиры-Спенсера $d \pi: T(S) \rightarrow T^{1}\left(Z_{0}\right)$ 
действует по формуле $d \pi(v)=\operatorname{cl}(v(F))$. Деформация $\pi$ называется регулярной, если росток $S$ неособый и композиция

$$
T(S) \stackrel{d \pi}{\longrightarrow} T^{1}\left(Z_{0}\right) \longrightarrow T^{1}\left(Z_{0}\right) / \mathfrak{m}^{2} T^{1}\left(Z_{0}\right)
$$

сюръективна, где $\mathfrak{m}$ обозначает максимальный идеал в алгебре $O_{0}(\Omega)$. Если $\pi$ регулярна, то пространство деформации $Z$ неособо.

Возьмем регулярную деформацию $\pi: Z \rightarrow S$, заданную вещественной голоморфной функцией $F$ (т.е. все производные функции $F$ в нуле вещественны). Ограничение этой деформации на вещественную часть $Z_{R}$ гиперповерхности $Z$ есть аналитическое отображение $\pi_{R}: Z_{R} \rightarrow S_{R}$, где $S_{R}:=S \cap \mathbb{R}^{n}$. Критическое множество отображения $\pi_{R}$ есть

$$
C_{R}(\pi):=\left\{F(s, \omega)=0, F_{\omega}^{\prime}(s, \omega)=0, s \in S_{R}, \omega \in \Omega_{R}:=\Omega \cap \mathbb{R}^{\nu}\right\} .
$$

Это неособое многообразие размерности $\operatorname{dim} S-1$. С деформацией связано симметричное лагранжево многообразие $\Lambda(\pi):=\Lambda_{+} \cup \Lambda_{-}$, где

$$
\Lambda_{ \pm, c}=\left\{\left(s, \xi= \pm F_{s}^{\prime}(s, \omega)\right),(s, \omega) \in C_{R}(\pi)\right\} .
$$

Многообразие $\Lambda(\pi)$ не имеет самопересечений, если окрестности $\Omega, S$ достаточно малы, а $\Lambda_{ \pm}$имеет кратность $\tau\left(Z_{0}\right)$ над началом координат.

Запишем поверхностный интеграл

$$
\delta_{F}(\rho, s):=\int_{Z_{s}} \frac{\rho}{d_{\omega} F(s, \cdot)}, \quad s \in S_{R} \backslash L,
$$

где $\rho$ - гладкая плотность с компактным носителем в $\Omega$, а $L-$ локус многообразия $\Lambda$. Под интегралом находится дифференциальная форма $\sigma$, такая, что $d_{\omega} F \wedge \sigma=\rho$. Она определена на множестве, где $d_{\omega} F \neq 0$, и интеграл не зависит от ее выбора. Поэтому функция (4.2) корректно определена на множестве $S_{R} \backslash L$; она может иметь особенности в $L$. Зафиксируем систему координат $s=\left(s_{0}, \ldots, s_{n}\right)$ в $S_{R}$ и положим $d s:=d s_{0} \wedge \cdots \wedge d s_{n}$.

ПреДлОЖенИЕ 4.1. Полуплотность $\delta_{F}(\rho, s) \sqrt{d s}$ является интегралом Фурье степени $(1-\nu) / 2$, лагранжево многообразие которого содержится в $\Lambda(\pi)$.

ДокАЗАТЕльство. Рассмотрим конус $\Theta:=\left\{\theta_{0} \neq 0\right\}$ во вспомогательном пространстве $\mathbb{R}^{N}, N=\nu+1$, с координатами $\theta_{0}, \ldots, \theta_{\nu}$ и центральную проекцию $r: \Theta \rightarrow \mathbb{R}^{\nu}$, которую мы запишем в виде $r(\theta)=\omega:=\theta_{0}^{-1}\left(\theta_{1}, \ldots, \theta_{\nu}\right)$. Функция $\phi(s, \theta)=\theta_{0} F(s, r(\theta))$ есть невырожденная фаза в конусе $\Theta$, поскольку деформация $\pi$ регулярна. Мы утверждаем, что $\delta_{F}(\rho, s)=I(\phi, a)$, где $a d \theta=d \theta_{0} \wedge r^{*}(\rho)$. Для проверки вычислим интеграл

$$
I(\phi, a)(s)=\int_{\Theta} \exp (2 \pi \imath \phi(s, \theta)) a(x, \theta) d \theta=\int_{\Omega_{R}}\left(\int \exp \left(2 \pi \imath F(s, \omega) \theta_{0}\right) d \theta_{0}\right) \rho .
$$

Внутренний интеграл равен дельта-функции $\delta_{F}$; следовательно, правая часть равна интегралу от частного $\rho / d_{\omega} F$ по $Z_{s}$, что и требовалось доказать. Чтобы вычислить степень интеграла Фурье $\delta_{F}(\rho, s) \sqrt{d s}$, мы положим $a:=\left|\theta_{0}\right|^{-\nu} r^{*}(\alpha)$, где $\rho=\alpha d \omega_{1} \wedge \cdots \wedge d \omega_{\nu}$. Здесь амплитуда $a$ однородна степени $-\nu$; следовательно, степень интеграла Фурье равна $N / 2-\nu=(1-\nu) / 2$.

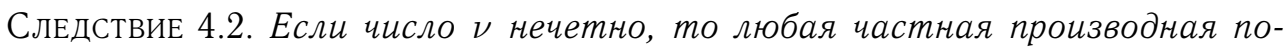
рядка $\kappa:=(\nu-1) / 2$ полуплотности $\delta_{F}(\rho, s) \sqrt{d s}$ есть интеграл Фурье cтепени 0. 


\section{§5. Эллиптические деформации}

Выберем систему координат $\omega_{1}, \ldots, \omega_{\nu}$ в $\Omega$ и предположим, что $f=f(\omega)$ есть квазиоднородный (взвешенно однородный) полином веса 1 с координатными весами $w_{1}, \ldots, w_{\nu}$. Эти веса подчинены условиям $0<w_{j} \leqslant 1 / 2$, определены однозначно и являются рациональными числами. Мы имеем $E_{\omega}(f)=f$ для эйлерова поля $E_{\omega}=\sum w_{j} \omega_{j} \partial_{\omega_{j}}$. Это векторное поле отталкивает от начала координат, поскольку все веса положительны. Полином $g$ квазиоднороден веса $w(g)$, если $E(g)=w(g) g$. Предположим, что деформация $\pi$ имеет вид

$$
F(s, \omega)=f(\omega)+s_{0} e_{0}(\omega)+\cdots+s_{n} e_{n}(\omega),
$$

где $e_{j}, j=0, \ldots, n,-$ некоторые квазиоднородные полиномы, образы которых в $T^{1}\left(Z_{0}\right)$ линейно независимы. Если эти образы составляют базис, то деформация (5.1) минимальная версальная. Чтобы сделать функцию $F$ квазиоднородной, мы присвоим вес $1-w\left(e_{j}\right)$ переменной $s_{j}, j=0, \ldots, n$. Координата $s_{j}$ в базе $S$ называется эллиптической, если ее вес положителен. Если вес равен нулю или отрицателен, то мы называем эту координату параболической или гиперболической соответственно. Рассмотрим эйлерово поле

$$
E_{s}:=\sum_{j}\left[1-w\left(e_{j}\right)\right] s_{j} \frac{\partial}{\partial s_{j}},
$$

определенное на базе $S$. Рассмотрим поток $\left\{g_{t}\right\}$, порожденный полем $E_{s}$, т.е. группу локальных диффеоморфизмов окрестности $S$, такую, что $\left.\partial_{t} g_{t}\right|_{t=0}=E_{s}$ и $g_{t} g_{r}=g_{t+r}$ для любых положительных $t, r$. Эллиптичность координаты $s_{j}$ означает, что $\left|s_{j}\right|$ возрастает под действием этого потока. Сделав линейную замену переменных $s_{0}, \ldots, s_{n}$, мы добьемся выполнения соотношений $e_{0}=1$, $e_{1}=\omega_{1}, \ldots, e_{\mu}=\omega_{\mu}, e_{j} \in \mathfrak{m}^{2}, j=\mu+1, \ldots, n$. Координаты $s_{0}, \ldots, s_{\mu}$ всегда эллиптичны, поскольку $w\left(e_{0}\right)=0, w\left(e_{j}\right)=w_{j} \leqslant 1 / 2, j=1, \ldots, \mu$.

ОПреДЕЛЕНИЕ. Мы называем деформацию $\pi$ эллиптической, если отображение $d \pi$ инъективно и все координаты в $S$ эллиптичны. Росток $Z_{0}$ называется эллиптическим, если его минимальная версальная деформация вида (5.1) эллиптична. Это означает, что $w(e)<1$ для любого монома, образ которого в $T^{1}\left(Z_{0}\right)$ не равен нулю. Особенности типов A, D, Е квазиоднородны и эллиптичны. Обратное утверждение следует из результата Саито [7].

Выберем регулярную эллиптическую деформацию и применим дельта-распределение (4.2) к произвольной квазиоднородной плотности $\rho$. Предположим сначала, что цикл $Z_{s}$ компактен при любом $s \in S_{R} \backslash L$. Тогда функция (4.2) определена и является гладкой в $S_{R} \backslash L$; она также квазиоднородна с весами $w\left(s_{j}\right), j=0, \ldots, n$. Производная $\partial / \partial s_{0}$ есть квазиоднородный оператор веса -1 ; следовательно, функция $V_{\rho}(s):=\left(\partial / \partial s_{0}\right)^{\kappa} \delta_{F}(\rho, s)$ имеет вес $w\left(\delta_{F}(\rho)\right)-\kappa=$ $w(\rho)-1-\kappa$, где $w(\rho)=w(\alpha)+w$ для однородной плотности $\rho=\alpha d \omega$, причем $d \omega:=d \omega_{1} \wedge \cdots \wedge d \omega_{\nu}, w:=w_{1}+\cdots+w_{\nu}$. Поэтому полуплотность $V_{\rho} \sqrt{d s}$ также квазиоднородна веса

$$
w\left(V_{\rho} \sqrt{d s}\right)=w(\rho)+\frac{1}{2}\left(\sum_{0}^{n}\left(1-w\left(e_{j}\right)\right)-1-\nu\right) .
$$


Если цикл $Z_{s}$ не компактен, мы вводим эту полуплотность посредством регуляризации:

$$
V_{\rho}:=\lim _{\varepsilon \rightarrow 0}\left(\partial / \partial s_{0}\right)^{\kappa}\left(\delta_{F}\left(\rho_{\varepsilon}\right)\right) .
$$

Здесь $\rho_{\varepsilon}:=\exp (-\varepsilon\{\omega\}) \rho,\{\omega\}=\left(\sum \omega_{i}^{2 a_{i}}+1\right)^{1 / b}$, а натуральные числа $b, a_{1}, \ldots, a_{\nu}$ выбраны таким образом, что $w_{i}=b / 2 a_{i}, i=1, \ldots, \nu$. Функция $\{\omega\}$ гладкая, невырожденная и асимптотически квазиоднородная веса 1 , и $\rho_{\varepsilon} \rightarrow \rho$, когда $\varepsilon \searrow 0$.

Теорема 5.1. Пусть $\pi-$ произвольная эллиптическая деформация квазиоднородного ростка. Тогда

(i) предел (5.3) существует для $\rho=d \omega$ и любой точки $s \in S_{R} \backslash L$,

(ii) функция $V_{\rho}$ квазиоднородная веса (5.2),

(iii) $V_{\rho}=\left(\partial / \partial s_{0}\right)^{\kappa} \delta_{F}(\sigma)+h$, где $h \in C^{\infty}\left(S_{0}\right)$ для некоторой окрестности $S_{0} \subset S$ начала координат, если плотность $\sigma \in \mathscr{D}\left(\Omega_{R}\right)$ совпадает с $\rho$ в окрестности нуля.

ДОКАЗАТЕЛЬСТвО. Рассмотрим интегралы

$$
I_{ \pm}^{\lambda}(\tau, s):=\int_{\Omega}(F(s, \omega) \pm 0 \imath)^{\lambda} \tau
$$

с произвольной гладкой быстро убывающей плотностью $\tau$. Они сходятся при любом $s \in S_{R} \backslash L$ и являются голоморфными функциями комплексного параметра $\lambda$. Мы продолжим эти интегралы для квазиоднородных плотностей с помощью приведенной ниже теоремы 5.2 .

Замечание. Интегралы $\delta_{F}(\rho, \cdot)$, отвечающие версальной деформации $\pi$, играют роль специальных функций в теории интегралов Фурье [4].

Теорема 5.2. Пусть снова $\pi$ - любая регулярная эллиптическая деформа-

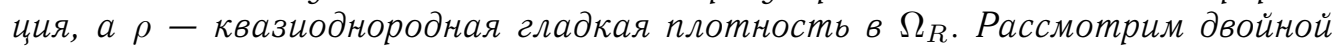
предел

$$
I_{ \pm}^{\lambda}(\rho, s):=\lim _{\varepsilon \searrow 0} \lim _{\delta \searrow 0} \int_{\Omega}(F(s, \omega) \pm \delta \imath)^{\lambda} \rho_{\varepsilon} .
$$

Если $\operatorname{Re} \lambda<-w(\rho)$, этот предел существует при $s \in S_{R} \backslash L$ и имеет мероморфное продолжение по параметру $\lambda$ с простыми полюсами в точках

$$
\lambda=-w(\rho)+\sum_{1}^{n} k_{j}\left(1-w\left(e_{j}\right)\right),
$$

где $k_{1}, \ldots, k_{n}$ - любые неотрицательные цеелье числа.

Доказательство содержится в [5] для случая эллиптических особенностей. Те же соображения применимы и для любой эллиптической деформации.

Вернемся к доказательству теоремы 5.1. Согласно теореме 5.2, все полюсы мероморфного продолжения принадлежат полуплоскости $\operatorname{Re} \lambda \geqslant-w(\rho)$. C другой стороны, для любой быстро убывающей плотности $\sigma$ справедливо соотношение

$$
\delta_{F}(\sigma)=\frac{1}{2 \pi \imath}\left(\int(F-0 \imath)^{-1} \sigma-\int(F+0 \imath)^{-1} \sigma\right),
$$

а $\partial / \partial s_{0}(F \pm 0 \imath)^{\lambda}=\lambda(F \pm 0 \imath)^{\lambda-1}$. Учитывая (5.3), мы определяем искомую специальную функцию в виде

$$
V_{\rho}(s):=(-1)^{\kappa} \frac{\kappa !}{2 \pi \imath}\left(I_{-}^{(-\kappa-1)}(\rho, s)-I_{+}^{(-\kappa-1)}(\rho, s)\right) .
$$


Точка $-\kappa-1$ не принадлежит указанной полуплоскости, так как $\kappa+1=(\nu+$ 1) $/ 2>\sum w_{j}=w(\rho)$. Поэтому правая часть корректно определена. Чтобы проверить п. (ii), мы заметим, что функции $I_{ \pm}^{\lambda}(\rho, \cdot)$ квазиоднородны веса $\lambda+w(\rho)$, т. е.

$$
E_{s}\left(I_{ \pm}^{\lambda}(\rho, s)\right)=(\lambda+w(\rho)) I_{ \pm}^{\lambda}(\rho, s)
$$

Это равенство легко проверяется при $\operatorname{Re} \lambda>0$ и, следовательно, выполняется всегда в силу единственности аналитического продолжения.

Докажем (iii). Если носитель плотности $\sigma$ не содержит особых точек слоя $Z_{s}$, то функции $I_{ \pm}^{\lambda}(\tau, \cdot)$ являются гладкими в окрестности точки $s$. С другой стороны, если точка $s$ близка к началу координат, то все особые точки гиперповерхности $Z_{s}$ близки к началу координат в $\Omega$. Это следует из эллиптичности деформации $\pi$. Поэтому носитель плотности $\rho_{\varepsilon}-\tau_{\varepsilon}=\exp (-\varepsilon\{\omega\})(\rho-\tau)$ не содержит особых точек этой гиперповерхности и функция $V_{\rho_{\varepsilon}}-\left(\partial / \partial s_{0}\right)^{\kappa}\left(\delta_{F}\left(\tau_{\varepsilon}\right)\right)$ является гладкой вблизи нуля. Переходя к пределу при $\varepsilon \searrow 0$, получаем (iii).

Вычислим контактный символ интеграла $V_{\rho}$. Выберем фазовую функцию $(5.1)$ в виде

$$
F(s, \omega):=f(\omega)+s_{0}+s_{1} \omega_{1}+\cdots+s_{\mu} \omega_{\mu}+s_{\mu+1} e_{\mu+1}(\omega)+\cdots+s_{n} e_{n}(\omega),
$$

где $e_{\mu+1}, \ldots, e_{n} \in \mathfrak{m}^{2}$. Положим $v=\left(\omega_{\mu+1}, \ldots, \omega_{\nu}\right)$ и заметим, что функции $\omega$, $\tilde{s}=\left(s_{\mu+1}, \ldots, s_{n}\right)$ образуют систему координат в $C_{R}(\pi)$. Мы можем использовать эту систему также на обеих частях $\Lambda_{ \pm}$при помощи отображений (4.1). Положим $d \tilde{s}=d s_{\mu+1} \wedge \cdots \wedge d s_{n}$.

СледствиЕ 5.3. Если деформация $\pi$ регулярна, то для любой формь $\tau=$ $\eta d \omega \in \mathscr{D}(\Omega)$ полуплотность $V_{\tau} \sqrt{d s}$ есть интеграл Фурье нулевой степени, причем $\Lambda(\phi) \subset \Lambda(\pi)$. Контактный символ этого интеграла равен образу полуплотности

$$
\sigma_{c}\left(V_{\tau}\right)=c_{\tau}(\tilde{s}, \omega) \eta \sqrt{\frac{ \pm d \tilde{s} \wedge d \omega}{\operatorname{det} f_{v v}^{\prime \prime}}}
$$

в многообразии $\Lambda(\pi)=\Lambda_{+} \cup \Lambda_{-}$, где $c_{\tau}-$ некоторая гладкая функция, такая, ито $\left|c_{\tau}(0,0)\right|=1$.

Доказательство следует из формул (2.3) и (2.4), поскольку $\partial F / \partial s_{0}=1$ и $\left|\theta_{0}\right|=c(\theta)|\theta|, c(0)=1$.

\section{§6. Энергетические показатели}

Пусть $Z_{0}-$ квазиоднородная гиперповерхность и $\pi: Z \rightarrow S-$ квазиоднородная версальная деформация ростка этой гиперповерхности в точке $z=0$. Рассмотрим подпространство $S_{e}$ в $S$, на котором обращаются в нуль все неэллиптические координаты. Мы назовем ограничение деформации $\pi$ на $S_{e}$ максимальной эллиптической деформацией ростка $Z_{0}$. Она определена однозначно с точностью до квазиоднородной биекции. Максимальная эллиптическая деформация всегда регулярна. 
ОПРЕДЕЛЕНИЕ. Пусть $\pi$ - максимальная эллиптическая деформация некоторого квазиоднородного ростка $Z_{0}$. Мы называем числа

$$
\begin{aligned}
e\left(Z_{0}\right) & :=2 w-\nu+\sum_{1}^{n}\left(1-w\left(e_{j}\right)\right), \quad w=\sum w_{j}, \\
e^{\prime}\left(Z_{0}\right) & :=\nu-2 w+\sum_{1}^{n}\left(1-w\left(e_{j}\right)\right),
\end{aligned}
$$

нижним и верхним энергетическими показателями ростка $Z_{0}$ соответственно. Оба показателя сохраняются при замене функции $f(\omega)$ на $f(\omega)+q(v)$, где $q-$ произвольная неособая квадратичная форма дополнительных координат $v$. Нижний показатель совпадает с весом распределения $\left|V_{\rho}\right|^{2} d s$ в силу формулы (5.2). Полином $\operatorname{det} f^{\prime \prime}$ квазиоднороден с весом $w(\eta)=\nu-2 w$ (максимальный вес в $\left.T^{1}\left(Z_{0}\right)\right)$. Для плотности $\tau=\operatorname{det} f^{\prime \prime} d \omega$ мы имеем формально

$$
w\left(\left|V_{\tau}\right|^{2} d s\right)=e\left(Z_{0}\right)+2 w(\eta)=\nu-2 w+\sum_{1}^{n}\left(1-w\left(e_{j}\right)\right)=e^{\prime}\left(Z_{0}\right),
$$

т. е. верхний показатель равен весу распределения $\left|V_{\tau}\right|^{2} d s$ при условии, что оно определено. Рассуждая, как в теореме 5.2, мы можем ее задать с помощью мероморфного продолжения интегралов $I_{ \pm}^{\lambda}$ в точку $\lambda=-\kappa-1$, если эта точка не является полюсом. Если она есть полюс, то его порядок равен 1 и мы определяем $V_{\tau}$ с помощью $(5.4)$, заменяя интегралы $I_{ \pm}^{\lambda}$ их вычетами в этой точке. В любом случае мы получаем некоторую обобщенную функцию $V_{\tau}$, причем распределение $\left|V_{\tau}\right|^{2} d s$ квазиоднородно с весом, равным верхнему энергетическому показателю. Оба распределения имеют вид плотностей энергии для сингулярных волновых полей $\delta_{F}(\rho), \delta_{F}(\tau)$ (в случае $n=3$ ); это служит мотивировкой термина энергетический.

С другой стороны, энергетические показатели совпадают с весами плотностей $d \tilde{s} \wedge d \omega$ и $\left|\operatorname{det} F_{\omega \omega}^{\prime \prime}\right|^{2} d \tilde{s} \wedge d \omega$ соответственно. Заметим при этом, что для деформации (5.1) матрица $F_{\omega \omega}^{\prime \prime}$ зависит только от переменных $\tilde{s}, \omega$. Ниже мы дадим интерпретацию этого совпадения в терминах геометрии критического множества $C_{R}(\pi)$.

СлЕДСТВИЕ 6.1. Для любой пробной плотности $\rho=\eta d \omega$

$$
\int_{D^{2} \geqslant \varepsilon^{2}} \alpha\left|V_{\rho}\right|^{2} d s=\left(\int_{C_{R}(\pi)} \frac{p^{*}(\alpha)|\eta|^{2}}{\operatorname{det} F_{v v}^{\prime \prime}} d \check{s} \wedge d \omega+o(1)\right) \log \frac{1}{\varepsilon}, \quad \varepsilon \rightarrow 0, \alpha \in \mathscr{D}(S),
$$

где $D$ есть барьер многообразия $\Lambda(\pi)$ в точке $0 \in S$.

ДоКАЗАТЕЛЬСтво. Это соотношение следует из теоремы 3.1, если мы подсчитаем символ интеграла $V_{\rho}$ с помощью следствия 5.3.

Поднимем поле $E_{s}$ до поля $E:=E_{\omega}+E_{s}$ в пространстве $\Omega \times S$. Поле $E$ касается $Z$, потому что $E(F)=F$. Пусть $E_{\Lambda}-$ образ эйлерова поля $E$ при диффеоморфизме $C_{R}(\pi) \cong \Lambda_{ \pm}(\pi)$, а $\left\{G_{t}, t>0\right\}-$ поток в $\Lambda(\pi)$, порожденный полем $E_{\Lambda}$. Выберем риманову метрику в $S_{R}$. Она порождает метрику в $T^{*}\left(S_{R}\right)$ и, следовательно, на $\Lambda(\pi)$. Для произвольного множества $K \subset \Lambda(\pi)$ мы положим $K_{t}:=G_{t}(K)$ и через $\operatorname{Vol}(K)$ обозначим риманов объем множества $K$. 
ПреДлОЖенИЕ 6.2. Пусть снова $\pi$ - максимальная эллиптическая деформация квазиоднородного ростка $Z_{0}$. Для любого компактного множества $K \subset \Lambda(\pi)$ мы имеем

$$
\begin{aligned}
\operatorname{Vol}\left(K_{t}\right) & =(c+o(1)) t^{e}, \\
\operatorname{Vol}\left(p\left(K_{t}\right)\right) & =\left(c^{\prime}+o(1)\right) t^{\left(e+e^{\prime}\right) / 2}
\end{aligned}
$$

при $t \rightarrow 0$, где е, $e^{\prime}$ суть энергетические показатели ростка $Z_{0}, c, c^{\prime}-$ некоторые положительные константы, а $p: \Lambda(\pi) \rightarrow S_{R}$ - естественная проекция.

ДокАЗАТЕЛЬСтво. Напомним, что эйлерово поле $E_{s}$ порождает некоторый поток $\left\{g_{t}\right\}$ в $S_{R}$. Для любой цепи $\gamma$, плотности $b$ и любой ограниченной функции $\alpha$ с компактным носителем мы имеем

$$
\int_{g_{t}(\gamma)} \alpha_{t} b=\int_{\gamma} \alpha g_{t}^{*}(b), \quad t>0
$$

где $\alpha_{t}=g_{1 / t}^{*}(\alpha)$. Выберем $\gamma=S(\varepsilon):=\left\{D^{2}(s) \geqslant \varepsilon\right\}$ и положим $b=\left|V_{\rho}\right|^{2} d s$, $\rho=d \omega$. В качестве барьера $D$ мы можем взять дискриминант $D(s)$ полинома $F$, который является квазиоднородным полиномом веса $m$. В этом случае $g_{t}((\varepsilon))=$ $\left\{D^{2}(s) \geqslant t^{m} \varepsilon\right\}=S\left(t^{m} \varepsilon\right)$. Тогда выполняется уравнение $g_{t}^{*}(b)=t^{e} b$, где $e-$ нижний показатель; следовательно,

$$
\int_{S\left(t^{m} \varepsilon\right)} \alpha_{t}\left|V_{\rho}\right|^{2} d s=t^{e} \int_{S(\varepsilon)} \alpha\left|V_{\rho}\right|^{2} d s
$$

Применим теорему 3.1 к обеим частям:

$$
\int_{\Lambda(\pi)} p^{*}\left(\alpha_{t}\right)\left|\sigma_{c}\right|^{2} \log \frac{1}{t^{m} \varepsilon}=(1+o(1)) t^{e} \int_{\Lambda(\pi)} p^{*}(\alpha)\left|\sigma_{c}\right|^{2} \log \frac{1}{\varepsilon}, \quad t \rightarrow 0 .
$$

Здесь $\sigma_{c}$ обозначает контактный символ интеграла $V_{\rho}$. С помощью следствия 5.3 мы получаем $\left|\sigma_{c}\right|^{2}=a(\tilde{s}, \omega) d \tilde{s} \wedge d \omega, a(0,0)=1$. Исключая логарифмические множители при $\varepsilon \rightarrow 0$, получаем

$$
\int_{C_{R}(\pi)} p^{*}\left(\alpha_{t}\right) d \tilde{s} \wedge d \omega=(1+o(1)) t^{e} \int_{C_{R}(\pi)} p^{*}(\alpha) d \tilde{s} \wedge d \omega, \quad t \rightarrow 0 .
$$

Мы имеем включение $s_{i} \mid C_{R}(\pi) \in \mathfrak{n}^{2}$ для $i=0, \ldots, \mu$, где $\mathfrak{n}$ обозначает максимальный идеал ростка $\left(C_{R}(\pi),(0,0)\right)$. Поэтому плотность $d \tilde{s} \wedge d \omega$ равна форме объема многообразия $C_{R}(\pi)$, умноженной на величину $c+o(1), c>0$, при $t \rightarrow 0$, и подобное утверждение верно для $\Lambda(\pi)$. Для проверки соотношения (6.1) мы можем предположить, что множество $K$ совпадает с $p^{-1}(p(K))$, поскольку проекция $p: \Lambda(\pi) / \mathbb{Z}_{2} \rightarrow L$ взаимно однозначна везде, за исключением подмножества многообразия $\Lambda(\pi)$ нулевой меры. Если функция $\alpha$ есть индикатор множества $p(K)$, то функция $p^{*}\left(\alpha_{t}\right)$ равна индикатору множества $K_{t}$; следовательно, левая часть формулы (6.3) равна объему компакта $K_{t}$ с коэффициентом $1+o(1)$. Это доказывает (6.1).

Для проверки соотношения (6.2) мы применяем вычетную форму к паре $V_{\tau}, V_{\rho}$, где $\rho=d \omega, \tau=\operatorname{det} F_{\omega \omega}^{\prime \prime} d \omega$. При этом мы можем предположить, что деформация 
задана формулой (5.5). Согласно следствию 5.3,

$$
\int_{D^{2}(s) \geqslant \varepsilon^{2}} V_{\tau} V_{\rho} d s=\left(\int_{C_{R}(\pi)} p^{*}(\alpha) \operatorname{det} F_{\omega \omega}^{\prime \prime} d \tilde{s} \wedge d \omega+o(1)\right) \log \frac{1}{\varepsilon} .
$$

Далее,

$$
\frac{\operatorname{det} F_{\omega \omega}^{\prime \prime}}{\operatorname{det} F_{v v}^{\prime \prime} \operatorname{det} F_{\omega \hat{s}}^{\prime \prime}} d \tilde{s} \wedge d \omega=(-1)^{m} p^{*}(d \hat{s} \wedge d \tilde{s}),
$$

где $\hat{s}=\left(s_{1}, \ldots, s_{\mu}\right)$ и $\operatorname{det} F_{\omega \hat{s}}^{\prime \prime}=1$ ввиду структуры формулы $(5.5)$. Поэтому

$$
\begin{aligned}
(-1)^{m} \int_{C_{R}(\pi)} p^{*}(\alpha) \frac{\operatorname{det} F_{\omega \omega}^{\prime \prime}}{\operatorname{det} F_{v v}^{\prime \prime} \operatorname{det} F_{\omega \hat{s}}^{\prime \prime}} d \tilde{s} \wedge d \omega & =\int_{C_{R}(\pi)} p^{*}(\alpha d \hat{s} \wedge d \tilde{s}) \\
& =\int_{L} \alpha d \hat{s} \wedge d \tilde{s}=\int_{p(K)} d \hat{s} \wedge d \tilde{s} .
\end{aligned}
$$

Затем мы повторяем предыдущие соображения и приходим к (6.2).

6.1. Вычисления и гипотезы. Оба энергетических показателя обращаются в нуль для ростка с невырожденной критической точкой, т. е. в случае $\tau\left(Z_{0}\right)=1$. Такие точки обеспечивают логарифмическую расходимость в теореме 3.1. В остальных случаях энергетические показатели строго положительны. Для эллиптических ростков мы имеем

$$
\begin{array}{rlrl}
e\left(A_{k}\right) & =\frac{k^{2}-k}{2(k+1)}, & e^{\prime}\left(A_{k}\right)=\frac{k^{2}+3 k-4}{2(k+1)}, & k \geqslant 1, \\
e\left(D_{k}\right)=\frac{k^{2}-4 k+6}{2(k-1)}, & e^{\prime}\left(D_{k}\right)=\frac{k^{2}-2}{2(k-1)}, & k \geqslant 4, \\
e\left(E_{k}\right)=\frac{k-1}{3}, & e^{\prime}\left(E_{k}\right)=\frac{10}{3}, \frac{34}{9}, \frac{63}{15}, & k=6,7,8 .
\end{array}
$$

Для параболических ростков $P_{k}(k$ обозначает число Тюриной $)$

$$
e\left(P_{k}\right)=\frac{k}{2}-1, \quad e^{\prime}\left(P_{k}\right)=\frac{k}{2}, \quad k=8,9,10 .
$$

Рассмотрим росток, заданный однородной функцией $f\left(\omega_{1}, \omega_{2}\right)=\omega_{1}^{m}+\omega_{2}^{m}, m>2$, для которого $\tau\left(Z_{0}\right)=(m-1)^{2}$. Максимальная эллиптическая деформация задается функцией

$$
\begin{gathered}
F\left(s ; \omega_{1}, \omega_{2}\right)=f\left(\omega_{1}, \omega_{2}\right)+\sum_{i+j<m-1} s_{i j} \omega_{1}^{i} \omega_{2}^{j}+\sum_{i+j=m-1, i j>0} s_{i j} \omega_{1}^{i} \omega_{2}^{j}, \\
e=\frac{2}{m}-3+\frac{(m+1)(m+2)}{6}, \quad e^{\prime}=e+4-\frac{8}{m} .
\end{gathered}
$$

Аналогично, для функции $f\left(\omega_{1}, \omega_{2}, \omega_{3}\right)=\omega_{1}^{m}+\omega_{2}^{m}+\omega_{3}^{m}, m>2$, мы имеем $\tau\left(Z_{0}\right)=(m-1)^{3}$ и

$$
e=\frac{3}{m}-4+\frac{(m+1)(m+2)(m+3)}{24}, \quad e^{\prime}=e+6-\frac{12}{m} .
$$

Это вычисления делают правдоподобными следующие гипотезы:

ГипотезА I (оценка сверху). Для любого квазиоднородного ростка $Z_{0}$

$$
e\left(Z_{0}\right)+e^{\prime}\left(Z_{0}\right)<\tau\left(Z_{0}\right):=\operatorname{dim} T^{1}\left(Z_{0}\right) .
$$


Это неравенство, очевидно, выполняется для любого ростка из предыдущих списков.

ГиПотЕзА II (полунепрерывность). Если $\left(Z^{\prime}, z\right)$ есть некоторый особый росток некоторого слоя версальной деформации некоторого квазиоднородного ростка $(Z, 0)$, причем точка z достаточно близка к 0 , то

$$
e^{\prime}\left(Z^{\prime}\right) \leqslant e^{\prime}(Z)
$$

Можно без труда проверить гипотезу II для эллиптических особенностей, отслеживая список «примыканий», имеющийся в [1]. Для однородных полиномов $f$ указанного выше вида обе гипотезы следуют из (6.4) и (6.5). Эти полиномы могут иметь более сложный вид; важно лишь, что число Тюриной ростка то же самое, что и для полинома указанного простейшего вида.

Отмечу, что если $\pi$ - деформация квазиоднородного ростка $Z_{0}$, а $M \subset S$ - модулярный страт, то уравнение $e\left(Z_{s}\right)=e(Z)$ выполняется в каждой точке $s \in M$, близкой к нулю. Действительно, мы имеем $\tau\left(Z_{s}\right)=\tau(Z)$; следовательно, росток $Z_{s}$ также квазиоднородный с теми же весами, откуда следует, что $e\left(Z_{s}\right)=$ $e(Z), e^{\prime}\left(Z_{s}\right)=e^{\prime}(Z)$.

Автор благодарит Б. Мартина и Т. Хирша за очень полезные обсуждения.

\section{ЛИТЕРАТУРА}

1. Арнольд В. И., Гусеин-Заде С. М., Варченко А. Н. Особенности дифференцируемых отображений. Т. 1. Наука, М., 1982.

2. Hörmander L. Fourier integral operators, I. Acta Math., 127, Nos. 1-2, 79-183 (1971).

3. Паламодов В. П. Асимптотические разложения интегралов в комплексной и вещественной областях. Матем. сб., 127, №2, 209-238 (1985) .

4. Паламодов В. П. Обобщенные функции и гармонический анализ. ВИНИТи. Т. 72 , 1991.

5. Palamodov V. P. Special functions of several variables. In: Linear topological spaces and complex analysis III. METU-Tubitak, Ankara, 1997, pp. 120-137.

6. Palamodov V. P. Dynamics of wave propagation and curvature of discriminants. Ann. Inst. Fourier, 50, No. 6, 1945-1981 (2000).

7. Saito K. Quasihomogene isolierte Singularitäten von Hyperflächen. Invent. Math., 14, 123-142 (1971).

Tel Aviv University e-mail:palamodo@post.tau.ac.il 24 декабря 1999 г. 